\title{
СРАВНЕНИЕ СКОРОСТИ СХОДИМОСТИ БЫСТРЫХ РАЗЛОЖЕНИЙ С РАЗЛОЖЕНИЯМИ В КЛАССИЧЕСКИЙ РЯД ФУРЬЕ
}

\author{
А. Д. Чернышов ${ }^{\star}$ В. В. Горяйнов ${ }^{\star \star}$, О. В. Лешонков ${ }^{\star \star *}$, \\ Е. А. Соболева ${ }^{\star}$ О. Ю. Никифорова \\ * Воронежский государственный университет инженерных технологий \\ **Воронежский государственный технический университет \\ ${ }^{* * *}$ АО «НИИЭТ» г. Воронеж
}

Поступила в редакцию 18.01.2019 г.

\begin{abstract}
Аннотация. Путем численного сравнения показано большое превосходство быстрых разложений по сравнению с разложениями в классический ряд Фурье. Эксперименты проводились для гладких функций, функций с участком резкого возрастания и для быстро осциллирующей функции. Данные сравнения показали, что для достижения одинаковой точности в некоторых случаях в быстрых разложениях достаточно учитывать примерно в 1000 раз меньше членов по сравнению с классическим рядом Фурье. Кроме того, быстрые разложения допускают определенное число раз почленное дифференцирование, тогда как классические ряды Фурье в общем случае не допускают подобное дифференцирование.

Ключевые слова: быстрые разложения, ряды Фурье, коэффициенты Фурье, вычислительный эксперимент, погрешность вычислений, скорость сходимости ряда.

Annotation. Numerical comparison shows the great superiority of fast expansions in comparison with expansions in the classical Fourier series. The experiments were carried out for smooth functions, functions with a sharp increase and for a rapidly oscillating function. Comparison data showed that in order to achieve the same accuracy, in some cases in fast decompositions it is sufficient to take into account about 1000 times less terms than the classical Fourier series. In addition, rapid expansions admit a finite number of term-by-term differentiation, whereas classical Fourier series in the general case do not admit such differentiation.

Keywords: fast expansions, Fourier series, of Fourier coefficients, computing experiment, an error of evaluations, a velocity of convergence of a series.
\end{abstract}

\section{ВВЕДЕНИЕ}

При моделировании ситуаций в различных областях системного анализа используются дифференциальные уравнения с различными свойствами: при описании систем массового обслуживания [1], при решении задач, связанных с распространением волн на пространственных сетях [2], задач радиоэлектроники [3], массообмена [4], механики $[5,6]$. Одним из эффективных инструментов решения подобных уравнений являются ряды Фурье [6, 7]. Как известно, данные ряды медленно сходятся к раскладываемой функ-

(C) Чернышов А. Д., Горяйнов В. В., Лешонков О. В., Соболева Е. А., Никифорова О. Ю., 2019 ции. Для улучшения сходимости рядов Фурье в работах [8-17] разработан и апробирован метод быстрых разложений.

\section{КРАТКИЕ СВЕДЕНИЯ О БЫСТРЫХ РАЗЛОЖЕНИЯХ}

Быстрым разложением функции $f(x)$ называется сумма специально сконструированной граничной функции $M(x)$ и ряда Фурье для разности $f(x)-M(x)$. Функция $M(x)$ наделяется такими свойствами, чтобы разность $f(x)-M(x)$ удовлетворяла однородным граничным условиям. Это свойство обеспечивает быструю сходимость, что позволяет учитывать небольшое количество членов в ряде Фурье и экономить время вычислений 


\section{А. Д. Черныпиов, В. В. Горяйнов, О. В. Лешонков, Е. А. Соболева, О. Ю. Никифорова}

на ЭВМ. В работе [9] предложены граничные функции для разложений по синусам при выполнении условий Дирихле, и по косинусам при выполнении условий Неймана. В общем случае быстрое синус-разложение записывается в виде

$$
\begin{aligned}
& f(x)=C h_{2 p}=M_{2 p}(x)+\sum_{m=1}^{\infty} b_{m} \sin m \pi x, \\
& \left(f(x), M_{2 p}(x)\right) \in C^{(2 p+1)}, x \in[0,1], \\
& \left(f(x), M_{2 p}(x)\right) \in C^{(2 p+2)}, x \in(0,1),
\end{aligned}
$$

где $C h_{2 p}$ - оператор быстрых разложений порядка $2 p, b_{m}$ - коэффициенты Фурье разности $f(x)-M_{2 p}(x), M_{2 p}(x)$ - граничная функция.

В работах $[8,12]$ приводится алгоритм построения граничных функций $M_{2 p}(x)$ и $M_{2 p-1}(x)$ при любых $p$. Например, при $p=0 \div 3$ имеем граничные функции нулевого, второго, четвертого и шестого порядков соответственно, которые принимают вид [9, $10,19]$

$$
\begin{gathered}
M_{0}(x)=f(0)(1-x)+f(1) x, \\
M_{2}(x)=M_{0}(x)+ \\
+f^{\prime \prime}(0)\left(\frac{x^{2}}{2}-\frac{x^{3}}{6}-\frac{x}{3}\right)+f^{\prime \prime}(1)\left(\frac{x^{3}}{6}-\frac{x}{6}\right), \\
M_{4}(x)=M_{2}(x)+ \\
+f^{(4)}(0)\left(\frac{x^{4}}{24}-\frac{x^{5}}{120}-\frac{x^{3}}{18}+\frac{x}{45}\right)+ \\
+f^{(4)}(1)\left(\frac{x^{5}}{120}-\frac{x^{3}}{36}+\frac{7 x}{360}\right), \\
M_{6}(x)=M_{4}(x)+ \\
+f^{(6)}(0)\left(\frac{x^{6}}{720}-\frac{x^{7}}{5040}-\frac{x^{5}}{360}+\frac{x^{3}}{270}-\frac{2 x}{945}\right)+ \\
+f^{(6)}(1)\left(\frac{x^{7}}{5040}-\frac{x^{5}}{720}+\frac{7 x^{3}}{2160}-\frac{31 x}{15120}\right) .
\end{gathered}
$$

С ростом р скорость сходимости ряда Фурье в (1) быстро возрастает. Доказательство сходимости и оценка остаточного члена ряда приведены в работе [9]. Для практического сравнения скорости сходимости и эффективности данных быстрых разложений ниже приведены примеры.
Ряд Фурье в (1) устроен так, что допускает почленное дифференцирование до производной $(2 p+2)$ порядка включительно. При этом ряды для производных до $(2 p+1)$ порядка включительно равномерно сходятся всюду при $x \in[0,1]$, а для производной $(2 p+2)$ порядка сходится при $x \in(0,1)$ и могут расходится на границах $x=0, x=1$.

Обычно в рядах (1) ограничиваются конечным числом слагаемых, т. е.

$$
f(x) \approx M_{2 p}(x)+\sum_{m=1}^{N} b_{m} \sin m \pi x .
$$

Коэффициенты $b_{m}$ для разложения (2) вычисляются по формуле

$$
b_{m}=2 \int_{0}^{1}\left(f(x)-M_{2 p}(x)\right) \cdot \sin (m \pi x) d x .
$$

Если же интегралы (3) «неберущиеся», то коэффициенты $b_{m}$ в разложении (2) можно определить поточечным способом [10, 1619], который заключается в том, что область $x \in[0,1]$ разбивается равномерно на $(N+1)$ промежутков $N$ внутренними расчетными точками и записывается равенство (2) в каждой расчетной точке. Получается система алгебраических уравнений относительно коэффициентов $b_{m}$ :

$$
\begin{gathered}
f\left(x_{r}\right)=M_{2 p}\left(x_{r}\right)+\sum_{m=1}^{N} b_{m} \sin m \pi x_{r}, \\
x_{r}=r h, h=\frac{1}{N+1}, r=1 \ldots N,
\end{gathered}
$$

где $N$ - количество неизвестных коэффициентов $b_{m}$. Значения $x=0$ и $x=1$ не используются, т. к. на концах отрезка уравнение (4) обращается в тождество. Здесь задача о вычислении коэффициентов $b_{m}$ сводится к решению линейной алгебраической системы (4), решение которой можно найти в явном виде по формуле

$$
b_{m}=\frac{\sum_{r=1}^{N}\left(f\left(x_{r}\right)-M_{2 p}\left(x_{r}\right)\right) \cdot \sin \left(m \pi x_{r}\right)}{\sum_{r=1}^{N} \sin ^{2}\left(m \pi x_{r}\right)} .
$$


Сравнение скорости сходимости быстрых разложений с разложениями в классический ряд Фурье

\section{РЕЗУЛЬТАТЫ И ИХ ОБСУЖДЕНИЕ}

Ниже приведены численные эксперименты сравнения разложений функций $x^{10}$, $\sin 1.2 \pi x, \sin 5.3 \pi x$ на отрезке $[0,1]$ в классический ряд Фурье и в быстрые разложения порядков 0, 2, 4, 6 с их точными значениями. Вычисления проводились с точностью до $\Delta \sim 10^{-3}$. Для классического ряда Фурье погрешность определялась в точке $x=0.98$, где погрешность существенная. В случае быстрых разложений в табл. 1, 2 и 3 приводится максимальная погрешность, которая может достигаться в разных точках отрезка $[0,1]$ и может быть уменьшена во много раз при увеличении $N$ - членов частичной суммы ряда Фурье или увеличении порядка $2 p$ граничной функции $M_{2 p}(x)$.

В табл. 1, 2, 3 обозначено $N_{p m}$ - количество членов учитываемых в ряде Фурье при использовании поточечного способа вычисления коэффициентов Фурье в быстром разложении, $N_{i n t}$ - количество членов учитываемых в ряде Фурье при использовании формулы (3) для вычисления коэффициентов Фурье в быстром разложении.

Из табл. 1 видно, что скорость сходимости классического ряда Фурье исследуемых функций гораздо меньше скорости сходимости быстрых разложений. Разница в количестве членов ряда Фурье при использовании классического ряда и быстрого разложения с оператором $C h_{0}$ нулевого порядка для функции $\sin 5.3 \pi x$ составляет $\left(N_{i n t}^{*}-N_{i n t}^{(0)}\right)=7926$ членов. Для функции $x^{10}$ эта разность равна $\left(N_{i n t}^{*}-N_{i n t}^{(0)}\right)=9954$, для $\sin 1.2 \pi x$ разность равна $\left(N_{i n t}^{*}-N_{i n t}^{(0)}\right)=5887$.
При использовании быстрого разложения с оператором $C h_{2}$ второго порядка для функции $\sin 5.3 \pi x$ разность между используемыми членами в рядах Фурье равна $\left(N_{i n t}^{*}-N_{i n t}^{(2)}\right)=$ $=7983$ члена, для функции $x^{10}$ подобная разность $\left(N_{i n t}^{*}-N_{\text {int }}^{(2)}\right)=9992$ и для функции $\sin 1.2 \pi x$ разность равна $\left(N_{i n t}^{*}-N_{i n t}^{(2)}\right)=5897$.

Если же в быстром разложении применяется оператор $C h_{4}$ четвертого порядка, то разность между используемыми членами в рядах Фурье для функции $\sin 5.3 \pi x$ составляет $\left(N_{i n t}^{*}-N_{i n t}^{(4)}\right)=7990$ членов, для функции $x^{10}$ данная разность $\left(N_{i n t}^{*}-N_{i n t}^{(4)}\right)=9996$ и для функции $\sin 1.2 \pi x$ разность составляет $\left(N_{\text {int }}^{*}-N_{\text {int }}^{(4)}\right)=5898$ членов.

Для быстрого разложения с оператором $C h_{6}$ шестого порядка разность между используемыми членами в рядах Фурье для функции $\sin 5.3 \pi x$ равна $\left(N_{\text {int }}^{*}-N_{\text {int }}^{(6)}\right)=7992$ члена, для функции $x^{10}$ эта разность составляет $\left(N_{i n t}^{*}-N_{i n t}^{(6)}\right)=9998$ и для функции $\sin 1.2 \pi x-$ $\left(N_{i n t}^{*}-N_{i n t}^{(6)}\right)=5899$.

Отсюда можно сделать вывод, что быстрые разложения во много раз превосходят классические ряды Фурье: для случая быстро осциллирующей функции до 1000 раз, для случая функции, имеющей особенность быстро возрастать до 5000 раз и для случая гладкой функции до 5900 раз.

Быстрые разложения с оператором $C h_{6}$ превосходят быстрые разложения с оператором $C h_{0}$ для случая быстро осциллирующей функции в 9 раз, для случая функции, имеющей особенность быстро возрастать в 23 раза и для случая гладкой функции в 13 раз.

В общем случае классический ряд Фурье не допускает почленное дифференцирова-

Количество членов ряда Фурье, необходимых для $\Delta_{\max }(f) \sim 10^{-3}$

\begin{tabular}{|c|c|c|c|c|c|c|c|c|c|}
\hline \multirow{3}{*}{$f(x)$} & Классический ряд Фурье & \multicolumn{4}{|c|}{ Быстрое разложение с граничной функцией } \\
\cline { 2 - 12 } & $x=0.98$ & \multicolumn{2}{|c|}{$M_{0}$} & \multicolumn{2}{|c|}{$M_{2}$} & \multicolumn{2}{|c|}{$M_{4}$} & \multicolumn{3}{|c|}{$M_{6}$} \\
\cline { 2 - 11 } & $N_{\text {int }}^{*}$ & $N_{p m}^{(0)}$ & $N_{\text {int }}^{(0)}$ & $N_{p m}^{(2)}$ & $N_{\text {int }}^{(2)}$ & $N_{p m}^{(4)}$ & $N_{i n t}^{(4)}$ & $N_{p m}^{(6)}$ & $N_{i n t}^{(6)}$ \\
\hline $\sin 5.3 \pi x$ & 8000 & 91 & 74 & 19 & 17 & 11 & 10 & 9 & 8 \\
\hline$x^{10}$ & 10000 & 57 & 46 & 9 & 8 & 4 & 4 & 2 & 2 \\
\hline $\sin 1.2 \pi x$ & 5900 & 16 & 13 & 3 & 3 & 2 & 2 & 1 & 1 \\
\hline
\end{tabular}




\section{А. Д. Черныпиов, В. В. Горяйнов, О. В. Лешонков, Е. А. Соболева, О. Ю. Никифорова}

ние, тогда как при использовании быстрого разложения подобное многократное почленное дифференцирование допустимо. По этой причине ниже в табл. 2 и 3 не приводятся данные с производными от функций, представленных классическим рядом Фурье.

Из табл. 2 видно, что с увеличением порядка граничной функции эффективность быстрого разложения для описания первой производной исследуемых функций существенно возрастает. Так, для поддержания точности $\Delta_{\max }\left(f^{\prime}\right) \sim 10^{-3}$ разница в количестве членов ряда Фурье при использовании быстрого разложения с оператором $C h_{0}$ и быстрого разложения с оператором $C h_{2}$ для первой производной функции $\sin 5.3 \pi x$ составляет $\left(N_{i n t, 1}^{(0)}-N_{i n t, 1}^{(2)}\right)=45326$ членов, для первой производной функции $x^{10}$ подобная разность равна $\left(N_{i n t, 1}^{(0)}-N_{i n t, 1}^{(2)}\right)=18168$ и для первой производной функции $\sin 1.2 \pi x$ разность составляет $\left(N_{i n t, 1}^{(0)}-N_{i n t, 1}^{(2)}\right)=1682$ члена.

Если же в быстром разложении применяется оператор $C h_{4}$, то разность $\left(N_{i n t, 1}^{(0)}-N_{i n t, 1}^{(4)}\right)$ между используемыми членами в рядах Фурье для первой производной функции $\sin 5.3 \pi x \quad$ составляет $\left(N_{i n t, 1}^{(0)}-N_{\text {int }, 1}^{(4)}\right)=45377$ членов, для первой производной функции $x^{10}$ разность равна $\left(N_{i n t, 1}^{(0)}-N_{i n t, 1}^{(4)}\right)=18192$ и для первой производной функции $\sin 1.2 \pi x-$ $\left(N_{\text {int }, 1}^{(0)}-N_{\text {int }, 1}^{(4)}\right)=1687$.

При использовании быстрого разложения с оператором $C h_{6}$ разность $\left(N_{i n t, 1}^{(0)}-N_{i n t, 1}^{(6)}\right)$ между используемыми членами в рядах Фурье для первой производной функции $\sin 5.3 \pi x \quad$ составляет $\left(N_{i n t, 1}^{(0)}-N_{i n t, 1}^{(6)}\right)=45386$ членов, для первой производной функции $x^{10}$ данная разность равна $\left(N_{i n t, 1}^{(0)}-N_{i n t, 1}^{(6)}\right)=18196$ и для первой производной функции $\sin 1.2 \pi x$ - $\left(N_{i n t, 1}^{(0)}-N_{i n t, 1}^{(6)}\right)=1688$.

Из (1) видно, что ряд Фурье после двукратного дифференцирования БР с граничной функцией $M_{0}$, будет расходящимся на границах отрезка $[0,1]$. По этой причине данные для второй производной быстрых разложений с $M_{0}$ в табл. 3 не приводятся.

Из табл. 3 видно, что скорость сходимости быстрого разложения с оператором $C h_{2}$ для вторых производных исследуемых функций гораздо меньше скорости сходимости быстрых разложений с операторами более высокого порядка. Разница $\left(N_{i n t, 2}^{(2)}-N_{i n t, 2}^{(4)}\right)$ в количестве членов ряда Фурье при использовании быстрого разложения с оператором $\mathrm{Ch}_{2}$ и быстрого разложения с оператором $C h_{4}$ для

Таблица 2

Количество членов ряда Фурье, необходимьх для $\Delta_{\max }\left(f^{\prime}\right) \sim 10^{-3}$

\begin{tabular}{|c|c|c|c|c|c|c|c|c|}
\hline \multirow{3}{*}{$f(x)$} & \multicolumn{6}{|c|}{ Быстрое разложение с граничной функцией } \\
\cline { 2 - 10 } & \multicolumn{2}{|c|}{$M_{0}$} & \multicolumn{2}{|c|}{$M_{2}$} & \multicolumn{2}{|c|}{$M_{4}$} & \multicolumn{2}{|c|}{$M_{6}$} \\
\cline { 2 - 9 } & $N_{p m, 1}^{(0)}$ & $N_{i n t, 1}^{(0)}$ & $N_{p m, 1}^{(2)}$ & $N_{i n t, 1}^{(2)}$ & $N_{p m, 1}^{(4)}$ & $N_{i n t, 1}^{(4)}$ & $N_{p m, 1}^{(6)}$ & $N_{i n t, 1}^{(6)}$ \\
\hline $\sin 5.3 \pi x$ & 55500 & 45400 & 85 & 74 & 25 & 23 & 15 & 14 \\
\hline$x^{10}$ & 22300 & 18200 & 36 & 32 & 9 & 8 & 4 & 4 \\
\hline $\sin 1.2 \pi x$ & 2070 & 1690 & 9 & 8 & 3 & 3 & 2 & 2 \\
\hline
\end{tabular}

Таблица 3

Количество членов ряда Фурье, необходимьх для $\Delta_{\max }\left(f^{\prime \prime}\right) \sim 10^{-3}$

\begin{tabular}{|c|c|c|c|c|c|c|}
\hline \multirow{2}{*}{$f(x)$} & \multicolumn{3}{|c|}{ Быстрое разложение с граничной функцией } \\
\cline { 2 - 7 } & \multicolumn{2}{|c|}{$M_{2}$} & \multicolumn{2}{|c|}{$M_{4}$} & \multicolumn{2}{c|}{$M_{6}$} \\
\cline { 2 - 7 } & $N_{p m, 2}^{(2)}$ & $N_{i n t, 2}^{(2)}$ & $N_{p m, 2}^{(4)}$ & $N_{i n t, 2}^{(4)}$ & $N_{p m, 2}^{(6)}$ & $N_{i n t, 2}^{(6)}$ \\
\hline $\sin 5.3 \pi x$ & 1380 & 1239 & 77 & 70 & 29 & 27 \\
\hline$x^{10}$ & 390 & 352 & 22 & 21 & 7 & 7 \\
\hline $\sin 1.2 \pi x$ & 60 & 53 & 6 & 6 & 3 & 3 \\
\hline
\end{tabular}


второй производной функции $\sin 5.3 \pi x$ составляет $\left(N_{i n t, 2}^{(2)}-N_{i n t, 2}^{(4)}\right)=1169$ членов, для второй производной $x^{10}$ разность равна $\left(N_{i n t, 2}^{(2)}-N_{i n t, 2}^{(4)}\right)=331$ и для второй производной функции $\sin 1.2 \pi x-\left(N_{i n t, 2}^{(2)}-N_{i n t, 2}^{(4)}\right)=47$.

Если же в быстром разложении применяется оператор $C h_{6}$, то разность $\left(N_{i n t, 2}^{(2)}-N_{i n t, 2}^{(6)}\right)$ между используемыми членами в рядах Фурье для второй производной функции $\sin 5.3 \pi x$ составляет $\left(N_{i n t, 2}^{(2)}-N_{i n t, 2}^{(6)}\right)=1212$ членов, для второй производной $x^{10}$ функции $\left(N_{i n t, 2}^{(2)}-N_{i n t, 2}^{(6)}\right)=345$ и для второй производной функции $\sin 1.2 \pi x-\left(N_{i n t, 2}^{(2)}-N_{i n t, 2}^{(6)}\right)=50$.

Отсюда можно сделать вывод, что для описания второй производной быстрое разложение с граничной функцией $M_{6}$ обладает самой большой скоростью сходимости из указанных в табл. 3.

Коэффициенты $b_{m}$ в ряде Фурье быстрых разложений можно вычислять по формулам Фурье (3) или поточечным способом по формуле (5). При этом в рядах Фурье используется $N_{p m}$ и $N_{i n t}$ членов. Сравнение скорости сходимости быстрых разложений, с различным способом вычисления коэффициентов Фурье $b_{m}$ будем проводить по разности $N_{p m}-N_{i n t}$, значения которой приведены в табл. 4. Если разность $N_{p m}-N_{i n t}$ большая, то предпочти- тельным способом определения коэффициентов Фурье является вычисление интегралами по формуле (3). Если значение $N_{p m}-N_{i n t}$ равно нулю или близко к нулю, то определение коэффициентов Фурье по формулам (3) и (5) можно считать эквивалентным. Из табл. 4 следует, что для быстрого разложения с граничной функции $M_{0}$ предпочтительным способом определения коэффициентов Фурье является вычисление по формуле (3), так как в этом случае разность $N_{p m}-N_{i n t}$ является существенной (особенно для первой производной), а для быстрого разложения с граничной функцией $M_{6}$ оба способа вычисления коэффициентов Фурье (по формулам (3) или (5)) будут эквивалентны.

\section{ЗАКЛЮЧЕНИЕ}

Для достижения одинаковой точности вычислений при использовании быстрых разложений достаточно учитывать примерно в 1000 раз меньше членов по сравнению с классическим рядом Фурье. При повышении порядка граничных функций преимущество быстрых разложений в скорости сходимости перед классическим рядом Фурье становится еще более значительным. Если гладкая функ-

Таблица 4

\begin{tabular}{|c|c|c|c|c|c|}
\hline \multicolumn{6}{|c|}{ Разность $N_{p m}-N_{\text {int }}$ nри $\Delta_{\max } \sim 10^{-3}$} \\
\hline \multirow{2}{*}{\multicolumn{2}{|c|}{ Функция и ее производные }} & \multicolumn{4}{|c|}{ Быстрое разложение с граничной функцией } \\
\hline & & $M_{0}$ & $M_{2}$ & $M_{4}$ & $M_{6}$ \\
\hline \multirow{3}{*}{$\sin 5.3 \pi x$} & $f(x)$ & 17 & 2 & 1 & 1 \\
\hline & $f^{\prime}(x)$ & 10100 & 11 & 2 & 1 \\
\hline & $f^{\prime \prime}(x)$ & Вычислять нельзя & 141 & 7 & 2 \\
\hline \multirow{3}{*}{$x^{10}$} & $f(x)$ & 11 & 1 & 0 & 0 \\
\hline & $f^{\prime}(x)$ & 4100 & 2 & 1 & 0 \\
\hline & $f^{\prime \prime}(x)$ & Вычислять нельзя & 38 & 1 & 0 \\
\hline \multirow{3}{*}{$\sin 1.2 \pi x$} & $f(x)$ & 3 & 0 & 0 & 0 \\
\hline & $f^{\prime}(x)$ & 380 & 1 & 0 & 0 \\
\hline & $f^{\prime \prime}(x)$ & Вычислять нельзя & 7 & 0 & 0 \\
\hline
\end{tabular}




\section{А. Д. Чернышов, В. В. Горяйнов, О. В. Лешонков, Е. А. Соболева, О. Ю. Никифорова}

ция не имеет участков с быстрым возрастанием или убыванием и не быстро осциллирует, то в ряде Фурье следует учитывать только $1 \div 3$ члена, остальные члены в ряде Фурье будут слишком малы. При этом вычисление коэффициентов Фурье с помощью интегралов по формуле (3) или поточечным методом по формуле (5) становятся эквивалентными. Для построения решения краевых задач метод быстрых разложений приводит к небольшим алгебраическим системам. Данные исключительные свойства быстрых разложений позволяют решать многие трудные задачи.

\section{СПИСОК ЛИТЕРАТУРЫ}

1. Катрахов, В. В. Асимптотический анализ СМО с бесконечным накопителем / В. В. Катрахов, Д. Е. Рыжков, Н. И. Головко // Вестник Воронеж. гос. ун-та. Сер. Системный анализ и информационные технологии. 2007. - № 1 - C. 139-148.

2. Копытин, А. В. Моделирование процесса распространения волн на сети / А. В. Копытин // Вестник Воронеж. гос. ун-та. Сер. Системный анализ и информационные технологии. - 2008. - №1 - С. 11-13.

3. Бирюк, Н. Д. Параметрическая система двух связных контуров с кондуктивной связью / Н. Д. Бирюк, Ю. Б. Нечаев, С. Ю. Алёхин // Вестник Воронеж. гос. ун-та. Сер. Системный анализ и информационные технологии. - 2008. - № 1. - С. 5-10.

4. Боровикова, М. М. Моделирование диффузии вещества в плоской случайно-неоднородной среде / М. М. Боровикова, В. Г. Задорожний // Вестник Воронеж. гос. ун-та. Сер. Системный анализ и информационные технологии. - 2006. - № 2. - С. 10-18.

5. Шашкин, А. И. Квазистатическое деформирование упругого стержня при продольном изгибе / А. И. Шашкин, Н. В. Минаева, А. В. Гриценко // Известия высших учебных заведений. Машиностроение. - 2008. - № 12. C. 21-25.

6. Тимошенко, С. П. Теория упругости / С. П. Тимошенко, Дж. Гудьер. - М. : Наука, 1979. - 560 c.
7. Казаков, А. Л. Построение модели неравномерного транспортного потока на примере железнодорожной грузовой станции / А. Л. Казаков // Современные технологии. Системный анализ. Моделирование. - 2009. № 3. - С. 27-32.

8. Чернышов, А. Д. Улучшенные ряды Фурье и граничные функции / А. Д. Чернышов // Актуальные проблемы прикладной математики, информатики и механики: сб. тр. междунар. конф. - Воронеж : ВГУ, 2009 г. - Ч. 2. С. 236-238.

9. Чернышов, А. Д. Метод быстрых разложений для решения нелинейных дифференциальных уравнений / А. Д. Чернышов // Журнал вычислительной математики и математической физики. - 2014. - Т. 54, № 1. C. 13-24.

10. Чернышиов, А. Д. О выборе оптимального порядка граничной функции в быстром разложении / А. Д. Чернышов, В.В.Горяйнов // Вестник Воронеж. гос. ун-та. Сер. Системный анализ и информационные технологии. - 2011. - № 1. С. 60-65.

11. Chernyshov, A. D. On the relationship between the number of points of inflection of the graph of the number of members Fourier series by using rapid expansions / A. D. Chernyshov, V. V. Gorjajnov // ISJ Theoretical \& Applied Science (ISPC Perspectives in science for 2016, Philadelphia, USA). - 2016. - № 1 (33). - P. 137141.

12. Чернышов, А. Д. Решение методом быстрых разложений задачи о сушке зерна / А. Д. Чернышов, И. О. Павлов, Е. В. Воронова, В. В. Горяйнов // Теплофизика и аэромеханика. - 2012. - Т. 19, № 6. - С. 739-749.

13. Чернышов, А. Д. Решение одного нелинейного интегро-дифференциального уравнения методом быстрых разложений / А. Д. Чернышов, В.В.Горяйнов // Вестник ЧГПУ им. И. Я. Яковлева. Серия: механика предельного состояния. - 2012. - № 4(12). C. $105-112$.

14. Чернышов, А. Д. Температурный режим при естественной конвекции термовязкой несжимаемой жидкости в емкости прямоугольной формы / А. Д. Чернышов, А. Н. Мар- 
ченко, В. В. Горяйнов // Тепловые процессы в технике. - 2012. - Т. 4, № 11. - С. 482-486.

15. Чернышов, А. Д. Исследование температурных полей в прямоугольной пластине с внутренним источником, зависящим от температуры, при помощи быстрых разложений / А. Д. Чернышов, В. В. Горяйнов, А. Н. Марченко // Теплофизика и аэромеханика. - 2016. - Т. 23, № 2. - С. 247-256.

16. Чернышов, А. Д. Применение метода быстрых разложений для расчета траекторий космических кораблей / А. Д. Чернышов, В. В. Горяйнов, О. А. Чернышов // Известия вузов. Авиационная техника. - 2015. - № 2. C. $41-47$.

17. Chernyshov, A. D. Analysis of the stress field in a wedge using the fast expansions with pointwise determined coefficients / A. D. Cher-

Чернышов Александр Данилович -д-р физ.мат. наук, профессор, профессор кафедры высшей математики и информационных технологий, ФГБОУВО «Воронежский государственный университет инженерных технологий» (ВГУИТ).

Тел.: (4732) 55-35-54 (рабочий),

89009636621 (домашний)

E-mail: chernyshovad@mail.ru

Горяйнов Виталий Валерьевич - канд. физ.мат. наук, доцент, доцент кафедры прикладной математики и механики, ФГБОУВО «Воронежский государственный технический университет» (ВГТУ).

Тел.: (4732) 71-53-62 (рабочий), 89507516621 (домашний)

E-mail: gorvit77@mail.ru

Лешонков Олег Владимирович - инженер АО «НИИЭТ» г. Воронеж.

Тел.: 89081450839 (домашний)

E-mail: leekripper@ya.ru nyshov, V. V. Goryainov, A. A. Danshin // IOP Conf. Series: Journal of Physics: Conf. Series 973. - 2018. - 012002. doi:10.1088/174265.

18. Чернышов, А. Д. О способе нанесения расчетных точек на отрезок при реализации поточечного метода вычисления коэффициентов быстрых разложений для решения краевой задачи с условиями Дирихле / А. Д. Чернышов, В. В. Горяйнов // Вестник Воронеж. гос. ун-та. Сер. Системный анализ и информационные технологии. - 2012. - № 2. - С. 30-35.

19. Горяйнов, В. В. Анализ погрешности быстрых рядов Фурье при их многократном дифференцировании для случая вычисления коэффициентов ряда поточечным методом / В. В. Горяйнов // Вестник Воронежского государственного технического университета. 2011. - T. 7, № 2. - C. 36-40.

Chernyshov Alexandr Danilovich - the doctor of physical and mathematical sciences, the professor, Voronezh State University of Engineering Technologies, Department of higher mathematics and information technology.

Tel.: (4732) 55-35-54

E-mail: chernyshovad@mail.ru

Goryainov Vitalij Valerevich - the candidate of physical and mathematical sciences, associate professor, Voronezh State Technical University, Department of Applied Mathematics and Mechanics.

Tel.: (4732) 71-53-62

E-mail: gorvit77@mail.ru

Leshonkov Oleg Vladimirovich - Engineer position Joint-stock company "NIIET", Vorinezh. Tel.: 89081450839 (home)

E-mail: leekripper@ya.ru 
А. Д. Чернышов, В. В. Горяйнов, О. В. Лешонков, Е. А. Соболева, О. Ю. Никифорова

Соболева Елена Александровна - канд. физ.- Soboleva Elena Alexsandrovna - the candidate мат. наук, доцент кафедры Высшей математики и информационных технологий ФГБОУ ВО «Воронежский государственный университет инженерных технологий». E-mail: sobol5661@yandex.ru

of physical and mathematical sciences, associate professor, Voronezh State University of Engineering Technologies, Department of higher mathematics and information technology.

Tel.: (4732) 55-35-54

E-mail: sobol5661@yandex.ru

Никифорова Ольга Юрьевна - старший преподаватель кафедры Высшей математики и информационных технологий ФГБОУ ВО «Воронежский государственный университет инженерных технологий». E-mail: niki22@mail.ru

Nikiforova Ol'ga Yu. - senior lecturer, Voronezh State University of Engineering Technologies, Department of higher mathematics and information technology.

Tel.: (4732) 55-35-54

E-mail: niki22@mail.ru 